\title{
Proposta de diretrizes brasileiras do exame e tratamento de retinopatia da prematuridade (ROP)
}

\author{
Brazilian guidelines proposal for screening and treatment of \\ retinopathy of prematurity (ROP)
}

\author{
Andrea Zin ${ }^{1}$ \\ Telma Florêncio ${ }^{2}$ \\ João Borges Fortes Filho ${ }^{3}$ \\ Célia Regina Nakanami ${ }^{4}$ \\ Nicole Gianini ${ }^{5}$ \\ Rosa Maria Graziano ${ }^{6}$ \\ Nilva Moraes ${ }^{7}$
}

Sociedade Brasileira de Pediatria, Sociedade Brasileira de Oftalmologia Pediátrica e Conselho Brasileiro de Oftalmologia

Em nome do Grupo de Trabalho ROP-Brasil.

Pós-Graduanda, nível doutorado, pelo Programa de Saúde da Criança e da Mulher, Instituto Fernandes Figueira FIOCRUZ - Rio de Janeiro (RJ) - Brasil; Pesquisadora do Departamento de Neonatologia do FIOCRUZ (MS) - Rio de Janeiro (RJ) - Brasil.

Departamento de Retina Clínica e Cirúrgica da Fundação Altino Ventura. Coordenadora do Projeto de Prevenção a Cegueira por ROP na cidade do Recife (PE) - Brasil. Professor de Oftalmologia na Faculdade de Medicina da Universidade Federal do Rio Grande do Sul - UFRS Porto Alegre (RS) - Brasil; Pós-graduando em Oftalmologia pela Universidade Federal de São Paulo - UNIFESP - São Paulo (SP) - Brasil.

${ }^{4}$ Pós-Graduanda em Oftalmologia pela UNIFESP - São Paulo (SP) - Brasil; Chefe do Setor de Oftalmopediatria do Departamento de Oftalmologia do Instituto da Visão da UNIFESP - São Paulo (SP) - Brasil.

Cooordenadora da Câmara Técnica de Neonatologia da Secretaria Municipal de Saúde do Rio de Janeiro, Chefe de Clínica do CETRIN, Coordenadora do Grupo de Trabalho de Prevenção da Cegueira Infantil da Sociedade Brasileira de Pediatria SBP - Rio de Janeiro (RJ) Brasil.

Doutora, Médica Assistente do Departamento de Oftalmologia e Berçário Anexo à Maternidade do Hospital das Clínicas da Universidade de São Paulo - USP - São Paulo (SP) - Brasil; Presidente do Departamento de Oftalmologia da Sociedade de Pediatria de São Paulo e Primeira Secretaria da Sociedade Brasileira de Oftalmologia Pediátrica do Conselho Brasileiro de Oftalmologia.

Pós-graduanda em Oftalmologia pela UNIFESP - São Paulo (SP) - Brasil; Chefe do Setor de Retina e Vítreo do Instituto da Visão da UNIFESP - São Paulo (SP) - Brasil.

Endereço para correspondência: Andrea Zin. Departamento de Neonatologia. Instituto Fernandes FigueiraIFF-FIOCRUZ. Av. Rui Barbosa, 716 - Rio de Janeiro (RJ) CEP 22250-020

E-mail: andrea_zin@yahoo.com.br

Recebido para publicação em 10.01.2007

Última versão recebida em 06.04.2007

Aprovação em 24.04.2007

Nota Editorial: Depois de concluída a análise do artigo sob sigilo editorial e com a anuência do Dr. Ricardo Themudo Lessa Waetge sobre a divulgação de seu nome como revisor, agradecemos sua participação neste processo.

\begin{tabular}{|l|}
\hline RESUMO \\
\hline A retinopatia da prematuridade é uma das principais causas de cegueira \\
na infância, havendo cerca de 50.000 crianças cegas pela doença em todo \\
o mundo. A deficiência visual causada pela retinopatia dá-se em função \\
de descolamento de retina tracional. O exame de rotina de prematuros \\
possibilita a identificação de formas graves da doença, cujo tratamento \\
por fotocoagulação ou crioterapia pode reduzir significativamente a \\
cegueira por retinopatia da prematuridade (ROP). A seguir serão apresen- \\
tadas as diretrizes brasileiras para exame de prematuros e tratamento \\
daqueles com a forma grave da retinopatia da prematuridade (ROP), \\
recomendadas pela Sociedade Brasileira de Pediatria, Conselho Brasilei- \\
ro de Oftalmologia e Sociedade Brasileira de Oftalmologia Pediátrica. \\
Essas diretrizes foram baseadas nos resultados do I Workshop de Reti- \\
nopatia da Prematuridade e apresentam os atributos para a implementação \\
de um programa eficiente de diagnóstico e tratamento.
\end{tabular}

Descritores: Retinopatia da prematuridade/diagnóstico; Retinopatia da prematuridade/cirurgia; Prematuro; Diretrizes para a prática clínica

\section{INTRODUÇÃO}

A retinopatia da prematuridade (ROP) é uma enfermidade vasoproliferativa secundária à vascularização inadequada da retina imatura dos recém-nascidos prematuros (RN). É uma das principais causas de cegueira prevenível na infância, sendo responsável por 50.000 crianças cegas em todo o mundo ${ }^{(1-2)}$. A proporção de cegueira causada por ROP é muito influenciada pelo nível de cuidado neonatal (disponibilidade de recursos humanos, equipamentos, acesso e qualidade de atendimento), assim como pela existência de programas eficazes de triagem e tratamento. Por consegüinte, existe uma grande variabilidade de ocorrência da doença em países desenvolvidos e em desenvolvimento ${ }^{(3)}$.

A Classificação Internacional da ROP (ICROP) definiu a doença de acordo com sua gravidade (estadiamentos 1-5), localização (zonas I-III) e extensão em horas (1-12 h), com ou sem doença plus (dilatação arteriolar e tortuosidade venosa), cuja presença seria um indicador de atividade da doença $^{(4-5)}$ (Figura 1 e Tabela 1). Mais recentemente foi publicada uma atualização dessa classificação (ICROP-revisited), sendo reconhecida uma forma grave de doença posterior, a delimitação da Zona I e a existência da doença pré-plus ${ }^{(6)}$.

O ICROP definiu a doença limiar pela presença de ROP estágio 3, localizado nas zonas I ou II com extensão de pelo menos 5 horas contínuas ou 8 horas intercaladas e com identificação da dilatação arteriolar e venosa 


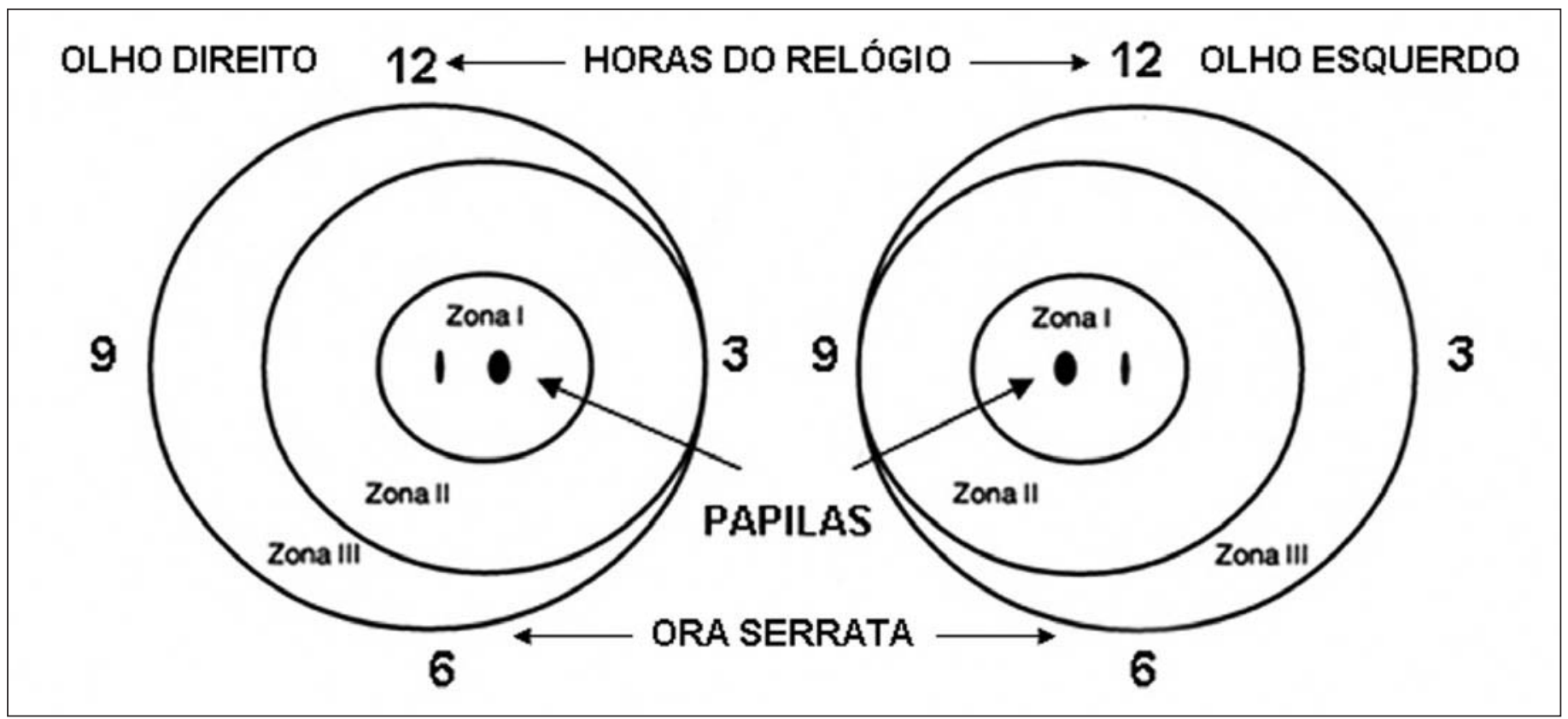

Figura 1 - Representação esquemática do fundo de olho

conhecida como doença plus. A significância clínica da doença limiar é que se o prematuro não for tratado neste momento terá chance de desenvolver complicações e resultados anatômicos e funcionais ruins em $50 \%$ dos $\operatorname{casos}^{(7)}$.

Os resultados do Multicenter Trial of Cryotherapy for Retinopathy of Prematurity Cooperative Group (CRYOROP) indicaram que o tratamento da doença limiar está associado à uma redução de $41 \%$ da ocorrência de pregas tracionais retinianas ou descolamento da retina e uma redução de 19-24\% na incidência de cegueira quando avaliados nos 5-15 anos subseqüentes. Contudo, apesar da disponibilidade do tratamento e de seu benefício indiscutível, mais de $40 \%$ das crianças permanecem com acuidade visual $<20 / 200$ no olho tratado aos 15 anos de idade ${ }^{(7-10)}$. Classicamente, de acordo com o CRYO-ROP, quando o diagnóstico de doença limiar era feito, o tratamento pela crioterapia ou laser estaria indicado. Em 2003, foram publicados os resultados do estudo Early Treatment for Retinopathy of Prematurity (ETROP). Foi demonstrado que o tratamento precoce, quando comparado ao convencional, está associado à uma redução no risco de baixa visão $(19,5 \%$ vs. $14,5 \% ; P=0,01)$ e de dano estrutural ao olho $(15,6 \% \text { vs. } 9,1 \% ; P<0,001)^{(11)}$ (Tabela 2$)$.

O tratamento precoce conforme o ETROP pode ser realizado na doença pré-limiar do tipo 1 (qualquer ROP em zona I com doença plus, estadiamento ROP 3, zona I mesmo sem doença plus e estadiamentos de ROP 2 ou 3 em zona II com doença plus) onde o risco de ocorrer um desfecho desfavorável é maior do que $15 \%{ }^{(11)}$.

A doença pré-limiar do tipo 2 ocorre quando se identifica a ROP em estadiamentos 1 ou 2, na zona I sem doença plus ou então em um estadiamento mais grave como ROP 3 na zona II mesmo sem a doença plus, onde a chance de ocorrer um desfecho anatômico e funcional desfavorável é menor do que $15 \%{ }^{(11)}$ (Tabela 1$)$.

\section{Quem deve ser examinado}

O desenvolvimento de programas de triagem para a identificação dos RN de risco que necessitem de tratamento é imprescindível para a redução da cegueira pela ROP.

Tabela 1. Classificação da retinopatia da prematuridade

\begin{tabular}{|c|c|}
\hline Estágio 1 & $\begin{array}{l}\text { Linha branca e plana que separa a } \\
\text { retina vascular da avascular }\end{array}$ \\
\hline Estágio 2 & Crista elevada \\
\hline Estágio 3 & $\begin{array}{l}\text { Proliferação fibrovascular a partir } \\
\text { da crista }\end{array}$ \\
\hline Estágio 4 & $\begin{array}{l}\text { A proliferação pode provocar um } \\
\text { descolamento de retina subtotal, (4a, } \\
\text { extrafoveal; } 4 \mathrm{~b} \text {, descolamento total, } \\
\text { incluindo fóvea) }\end{array}$ \\
\hline Estágio 5 & $\begin{array}{l}\text { Descolamento total de retina (funil } \\
\text { aberto ou fechado) }\end{array}$ \\
\hline $\begin{array}{l}\text { Doença limiar (definido pelo } \\
\text { CRYO-ROP) (se não tratada } \\
\text { pode apresentar resultados } \\
\text { anatômicos ruins em } 50 \% \\
\text { dos casos) }\end{array}$ & $\begin{array}{l}\text { Retinopatia estágio } 3 \text {, em zona I ou } \\
\text { II, com pelo menos } 5 \text { horas de ex- } \\
\text { tensão contínuas ou } 8 \text { horas inter- } \\
\text { caladas, na presença de doença } \\
\text { "plus" (dilatação arteriolar e veno- } \\
\text { dilatação) }\end{array}$ \\
\hline $\begin{array}{l}\text { Doença pré-limiar tipo } 1 \\
\text { (definido pelo ET-ROP) }\end{array}$ & $\begin{array}{l}\text { Qualquer ROP em zona I com plus } \\
\text { (doença posterior agressiva) } \\
\text { Estágio } 3 \text {, zona I, sem plus } \\
\text { Estágio } 2 \text { ou } 3 \mathrm{em} \text { zona II, com plus }\end{array}$ \\
\hline $\begin{array}{l}\text { Doença pré-limiar tipo } 2 \\
\text { (definido pelo ET-ROP) }\end{array}$ & $\begin{array}{l}\text { Estágio } 1 \text { ou } 2 \text {, zona I, sem plus } \\
\text { Estágio } 3 \text {, zona } 2 \text {, sem plus }\end{array}$ \\
\hline
\end{tabular}


Tabela 2. Indicação de tratamento

Pré-limiar tipo 1 (ETROP): Preferencial

Zona 1: qualquer estágio com plus

Zona 1: estágio 3

Zona 2: ROP 2 ou 3 com plus

Doença limiar (CRYO-ROP): não mais a única indicação

Retinopatia estágio 3, em zona I ou II, com pelo menos 5 horas de

extensão contínuas ou 8 horas intercaladas, na presença de doença plus

Vários são os critérios adotados para a seleção de pacientes para exame, contudo os mais conhecidos são:

1. American Academy of Pediatrics, American Academy of Ophthalmology e American Association for Pediatric Ophthalmology and Strabismus ${ }^{(12)}$ :

a. Peso de nascimento $(\mathrm{PN})<1.500$ gramas ou idade gestacional (IG) $\leq 30$ semanas, ou;

b. PN entre 1.500-2.000 gramas ou IG $\leq 30$ semanas com curso clínico instável (incluindo aqueles que necessitam de suporte cardio-respiratório e os que estejam em risco segundo critério do neonatologista);

c. Primeiro exame será baseado na IG ao nascimento: entre 31-36 semanas de idade corrigida para IG 22-32 semanas.

2. Royal College of Ophthalmologists e British Association of Perinatal Medicine $e^{(13)}$ :

a. $\mathrm{PN}<1.500$ gramas ou $\mathrm{IG}<31$ semanas;

b. Primeiro exame entre $6^{\underline{a}}$ e $7^{\underline{a}}$ semana de nascimento.

3. Canadian Association of Pediatric Ophthalmologists Ad Hoc Committee on Standards of Screening Examination for Retinopathy of Prematurity ${ }^{(14)}$ :

a. $\mathrm{PN} \leq 1.500$ gramas, $\mathrm{IG} \leq 30$ semanas;

b. Primeiro exame entre 4-6 semanas após o nascimento.

Contudo, as diretrizes utilizadas nos Estados Unidos, Canadá e Reino Unido podem não ser adequadas para a detecção dos prematuros em risco de desenvolver ROP grave e conseqüente deficiência visual ${ }^{(1-2)}$ em países nos quais os níveis de cuidados neonatais não sejam equivalentes. Com o objetivo de delinear o perfil dos $\mathrm{RN}$ de risco que apresentam ROP limiar em nosso país foi realizado o I Workshop de ROP, no Rio de Janeiro, em outubro de 2002, com o suporte do Programa Visão 2020-Agência Internacional de Prevenção da Cegueira (IAPB). Foi organizado pela IAPB, Instituto Vidi, Conselho Brasileiro de Oftalmologia (CBO), Sociedade Brasileira de Oftalmologia Pediátrica (SBOP) e Sociedade Brasileira de Pediatria (SBP) ${ }^{(15)}$. Sessenta e sete participantes de 17 cidades compareceram, entre pediatras e oftalmologistas, com o intuito de discutir a situação da ROP no Brasil.

Países com economias em desenvolvimento e que estão implementando ou expandindo serviços de tratamento intensivo neonatal nos setores público e privado parecem apresentar um maior percentual de cegueira infantil em função da falta de adequada triagem neonatal na busca da doença, estimando-se que das 100.000 crianças cegas na América Latina, 24.000 assim o são em decorrência da $\operatorname{ROP}^{(16)}$.

\section{Recomendações}

De acordo com os dados expostos no I Workshop de ROP, a Sociedade Brasileira de Pediatria, o Conselho Brasileiro de Oftalmologia e a Sociedade Brasileira de Oftalmologia Pediátrica recomendam as seguintes diretrizes a serem adotadas em todas as unidades neonatais do país:

\section{Exame}

1. Critérios de exame:

a. $\mathrm{PN} \leq 1.500$ gramas e/ou $\mathrm{IG} \leq 32$ semanas;

b. Considerar o exame em RNs com presença de fatores de risco:

i. Síndrome do desconforto respiratório;

ii. Sepse;

iii. Transfusões sangüíneas;

iv. Gestação múltipla;

v. Hemorragia intraventricular.

c. Primeiro exame: entre a $4^{\underline{a}}$ e $6^{\underline{a}}$ semana de vida;

d. Deve-se ter atenção no sentido de reduzir o desconforto e efeitos sistêmicos causados pela realização do exame: instilação de colírio anestésico, auxílio de uma enfermeira ou auxiliar de enfermagem para conter o prematuro, assim como considerar o uso de glicose durante o exame.

2. O exame deve ser realizado por oftalmologista com experiência em exame de mapeamento de retina em prematuros e conhecimento da doença para identificar a localização e as alterações retinianas seqüenciais, utilizando o oftalmoscópio binocular indireto. Devemos utilizar como referência The International Classification of Retinopathy of Prematurity Revisited ${ }^{(6)}$;

3. Deve-se prestar especial atenção para a zona de ocorrência da doença no fundo de olho e a presença de doença plus que irão determinar a necessidade de tratamento ou não;

4. O agendamento dos exames subseqüentes deverá ser determinado pelos achados do primeiro exame:

a. Retina madura (vascularização completa): seguimento com 6 meses (avaliação do desenvolvimento visual funcional, estrabismo, ametropias). Prematuros apresentam $46 \%$ de chance de apresentarem alguma dessas alterações oftalmológicas ${ }^{(17)}$;

b. Retina imatura (vascularização não completa) ou presença de ROP < pré-limiar: avaliação de 2/2 semanas;

c. Retinopatia em regressão: avaliação de 2/2 semanas;

d. Retina imatura, zona I: exames semanais;

e. ROP pré-limiar tipo $2^{(11)}$ : exames 3-7 dias;

f. ROP pré-limiar tipo 1 (zona 1, qualquer estágio com plus; zona I, estágio 3; zona II, estágio 2 ou 3 plus $)^{(11)} \mathrm{e}$ limiar: tratamento em até 72 horas;

g. Os exames podem ser suspensos quando a vascularização da retina estiver completa, idade gestacional corrigida de 45 semanas e ausência de ROP pré-limiar, ROP completamente regredida;

h. Recomenda-se a utilização de um formulário padrão (Anexo1). 


\section{Anexo 1 - Formulário de exame - Retinopatia da prematuridade - Grupo ROP Brasil}

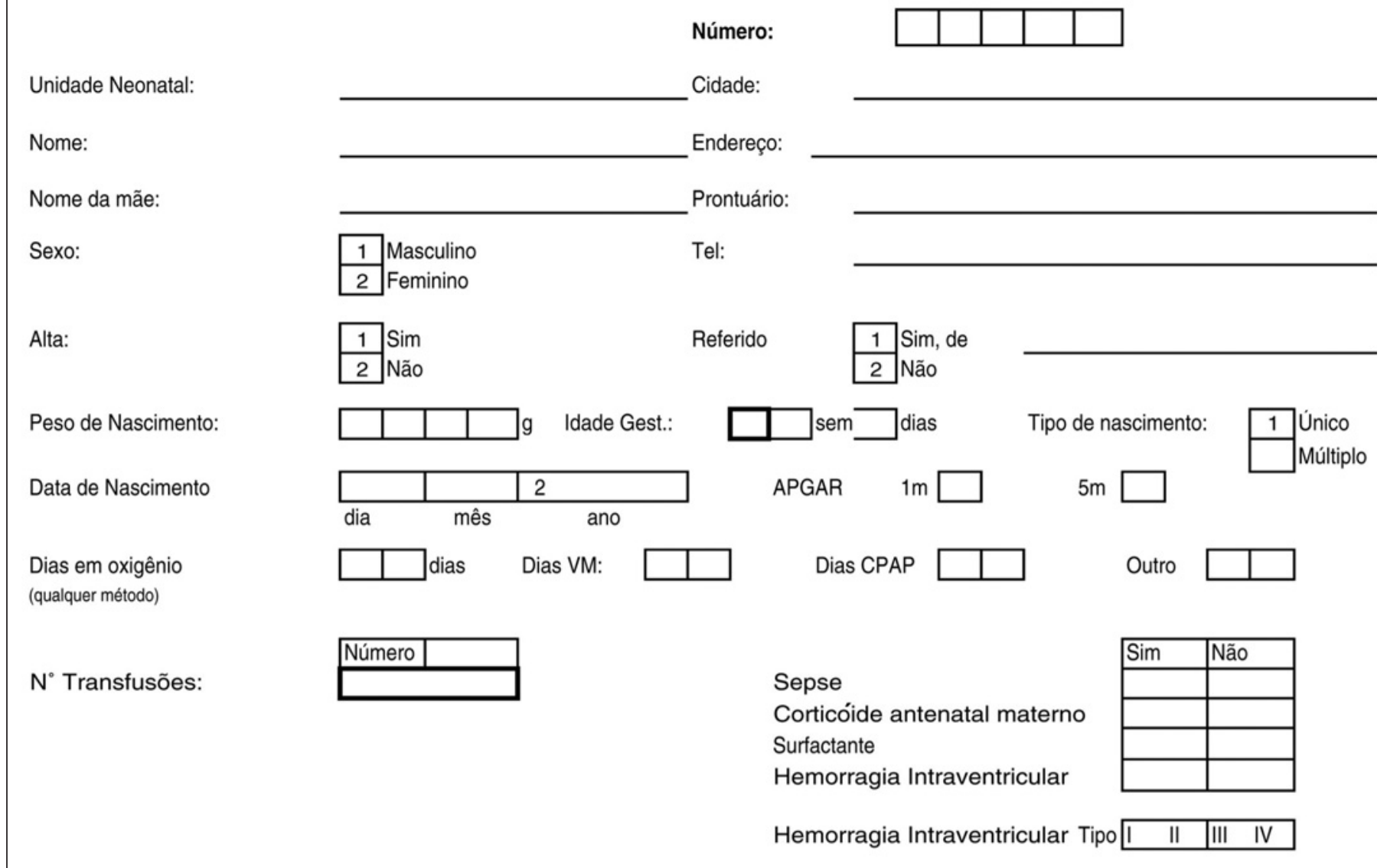

\section{Exame 1}

Data

I G Corrigida (sem)

Dilatação vasos íris (Sim ou Não)

Turvação vítrea (Sim ou Não)

Preplus (Sim ou Não)

Plus (Sim ou Não)

Estágio (0 a 5)

Zona (0 a 3)

Horas (0 a 12)
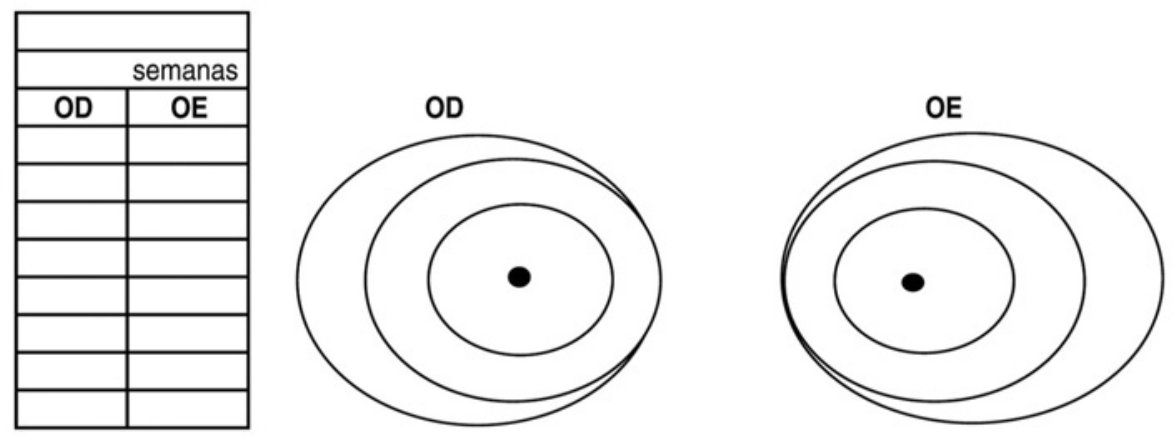

ROP Tipo 1 (Sim ou Não)

ROP Tipo 2 (Sim ou Não)

Limiar (sim ou não)

Em regressão

Regredido

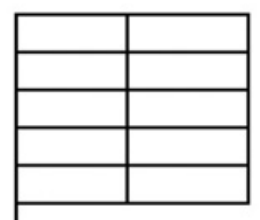

Conduta

Examinador:

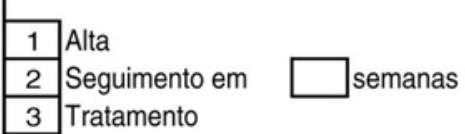

3 Tratamento
Sepse

Surfactante

Hemorragia Intraventricular Tipo I II 


\section{Continuação - Anexo 1 - Formulário de exame - Retinopatia da prematuridade - Grupo ROP Brasil} Nome

\section{Exame 2}

Data

I G Corrigida (sem)

Dilatação vasos íris (Sim ou Não)

Turvação vítrea (Sim ou Não)

Preplus (Sim ou Não)

Plus (Sim ou Não)

Estágio (0 a 5)

Zona (0 a 3)

Horas (0 a 12)

ROP Tipo 1 (Sim ou Não)

ROP Tipo 2 (Sim ou Não)

Limiar (sim ou não)

Em regressão

Regredido
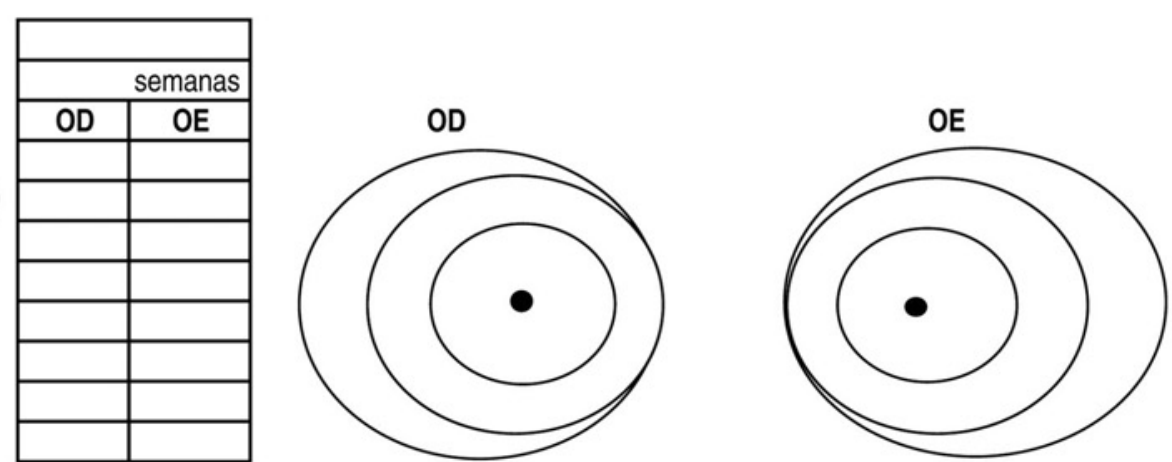

Conduta

Examinador:
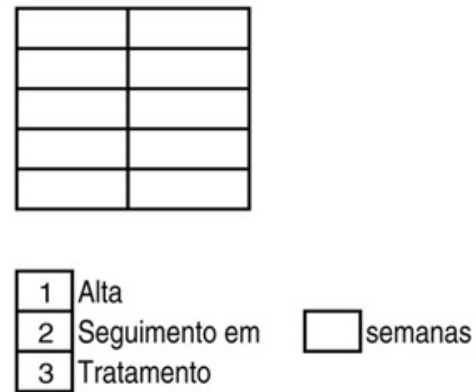

Data do próximo exame:

Nome

Exame 3

Data

I G Corrigida (sem)

Dilatação vasos íris (Sim ou Não)

Turvação vítrea (Sim ou Não)

Preplus (Sim ou Não)

Plus (Sim ou Não)

Estágio (0 a 5)

Zona (0 a 3)

Horas (0 a 12)

ROP Tipo 1 (Sim ou Não) ROP Tipo 2 (Sim ou Não)

Limiar (sim ou não)

Em regressão

Regredido
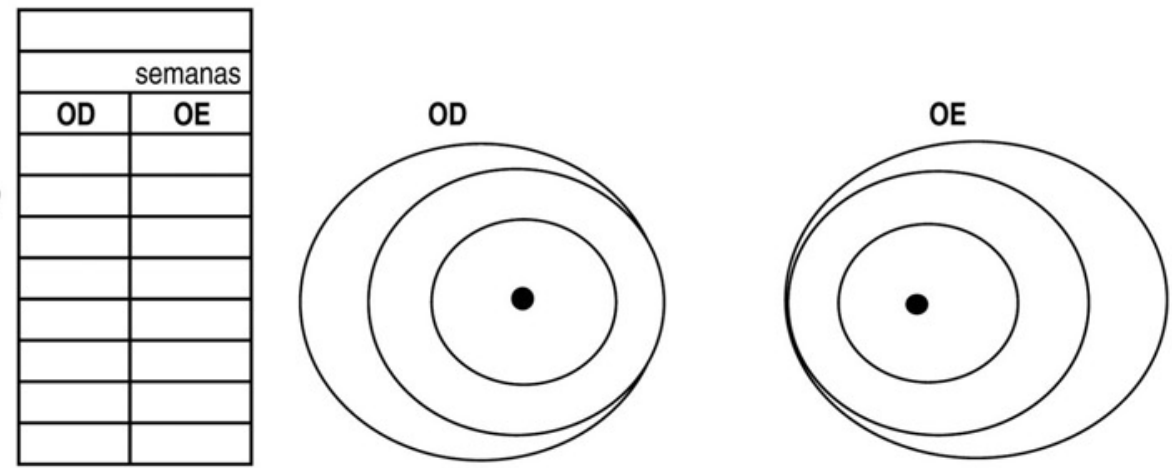

Conduta

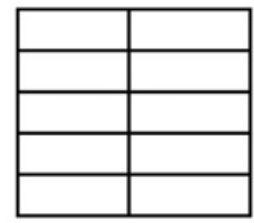

Prontuário/Hospital/Cidade:
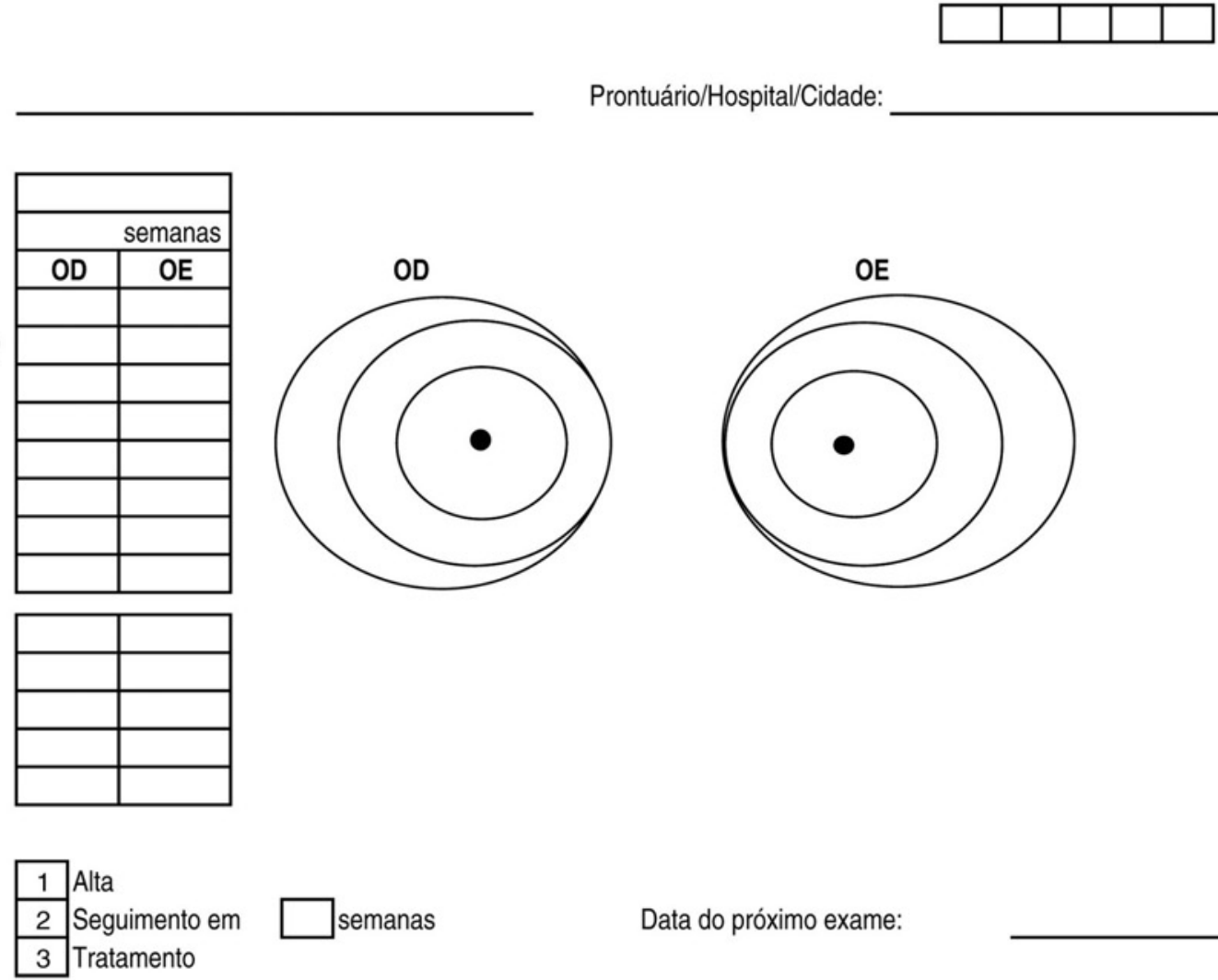

Data do próximo exame:

Examinador: 


\section{Continuação - Anexo 1 - Formulário de exame - Retinopatia da prematuridade - Grupo ROP Brasil}

\section{Exame 4}

Data

I G corrigida

Dilatação vasos íris (Sim ou Não)

Turvação vítrea (Sim ou Não)

Preplus (Sim ou Não)

Plus (Sim ou Não)

Estágio ( 0 a 5)

Zona (0 a 3)

Horas (0 a 12)
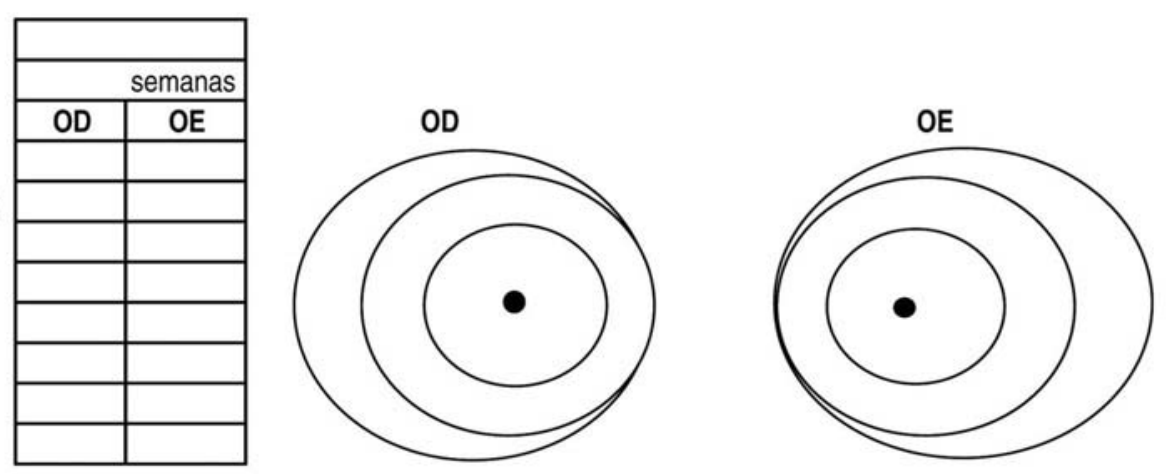

ROP Tipo 1 (Sim ou Não)

ROP Tipo 2 (Sim ou Não)

Limiar (sim ou não)

Em regressão

Regredido

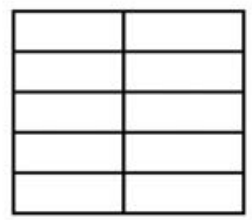

Conduta

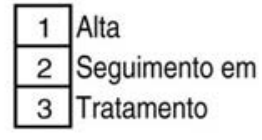

$\square$ semanas

Data do próximo exame:

Examinador:

\section{Exame 5}

Data

IG Corrigida

Dilatação vasos íris (Sim ou Não)

Turvação vítrea (Sim ou Não)

Preplus (Sim ou Não)

Plus (Sim ou Não)

Estágio ( 0 a 5)

Zona (0 a 3)

Horas (0 a 12)
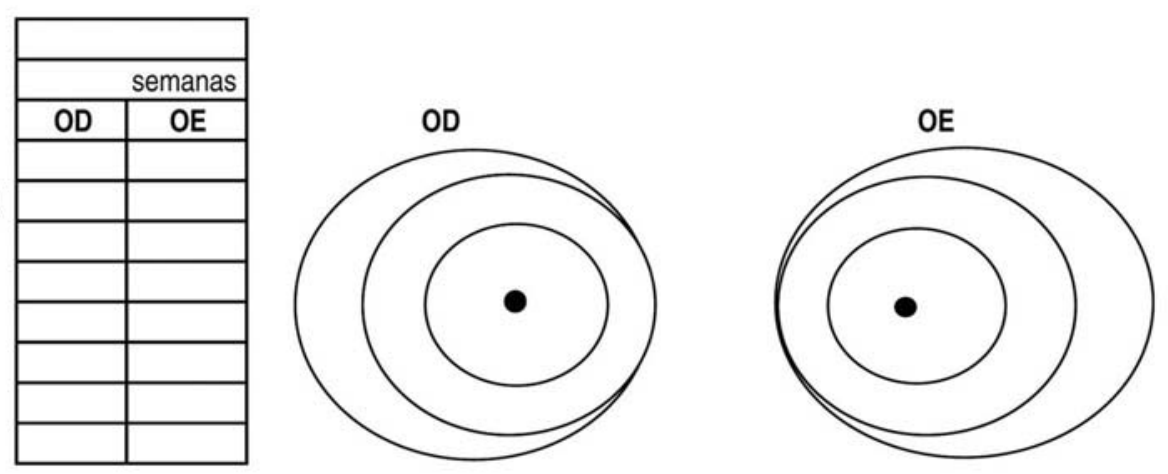

ROP Tipo 1 (Sim ou Não)

ROP Tipo 2 (Sim ou Não)

Limiar (sim ou não)

Em regressão

Regredido

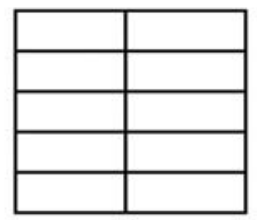

Conduta

\begin{tabular}{|l|l}
\hline 1 & Alta \\
\cline { 1 - 1 } 2 & Seguimento em \\
\cline { 1 - 1 } 3 & Tratamento \\
\cline { 1 - 1 } &
\end{tabular}

$\square$ semanas

Data do próximo exame:

Examinador: 


\section{Tratamento e Resultados Finais}

Nome

Olhos tratados

Diagnóstico pré tratamento:

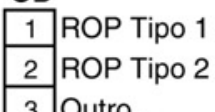

Método - 10 tto

\section{Laser apenas, número de spots:}

2 Crio apenas

3 Laser + crio

Data 10 tto

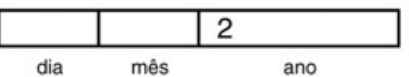

Tratado por:

1A VISITA PÓS-OP:

Data

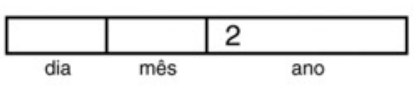

Semanas após tratamento

Achados

Data último exame:

\section{OD}

\begin{tabular}{|l|l}
\hline 1 & Regressão \\
\cline { 1 - 1 } 2 & Atividade da doença \\
\cline { 1 - 1 } 3 & Estágio 4a \\
\cline { 1 - 1 } 4 & Estágio 4b \\
\cline { 1 - 1 } 5 & Estágio 5 \\
\cline { 1 - 1 } 6 & Alts cicatriciais \\
\cline { 1 - 1 } 7 & Outros \\
\cline { 1 - 1 } 8 & Não compareceu \\
\hline
\end{tabular}

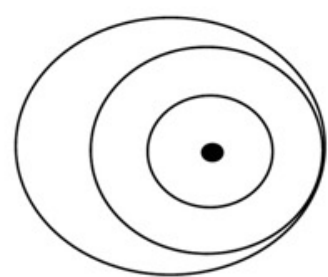

Necessário mais tto

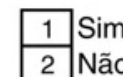

Data:

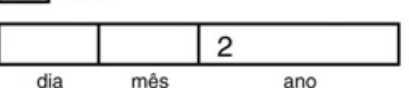

Resultado Final :

\begin{tabular}{|l|l}
\hline 1 & Regressão \\
\cline { 1 - 1 } 2 & Atividade da doença \\
\cline { 1 - 1 } 3 & Estágio 4a \\
\cline { 1 - 1 } 4 & Estágio 4b \\
\cline { 1 - 1 } 5 & Estágio 5 \\
\cline { 1 - 1 } 6 & Alts cicatriciais \\
\cline { 1 - 1 } 7 &
\end{tabular}

7 Outros

Encaminhado p/ oftalmol:

Comentários:

\begin{tabular}{|l|ll}
\hline 1 & Sim Onde e dia: \\
\hline 2 & Não & \\
\hline
\end{tabular}

Prontuário/Unidade/Cidade:

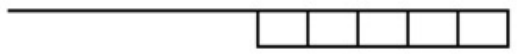

\section{OE}
1 ROP Tipo 1
2 ROP Tipo 2
3 Outro,

1 Laser apenas, número de spots:

3 Laser + crio

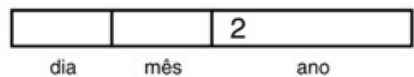

dia mês ano

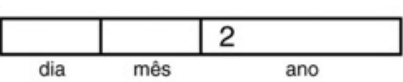

\section{$\mathrm{OE}$}

\begin{tabular}{|l|l}
\hline 1 & Regressão \\
\cline { 1 - 1 } 2 & Atividade da doença \\
\cline { 1 - 1 } 3 & Estágio 4a \\
\cline { 1 - 1 } 4 & Estágio 4b \\
\cline { 1 - 1 } 5 & Estágio 5 \\
\cline { 1 - 1 } 6 & Alts cicatriciais \\
\cline { 1 - 1 } 7 & Outros \\
\hline 8 & Não compareceu \\
\hline
\end{tabular}

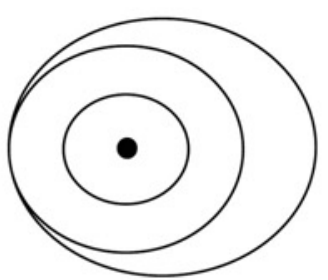

Data:
1 Regressão
2 Atividade da doença
3 Estágio $4 a$
4 Estágio 4b
5 Estágio 5
6 Alts cicatriciais
7 Outros

Examinado por: 
5. Os pais das crianças que apresentam ROP devem ser informados da natureza do problema e suas possíveis conseqüências, além da necessidade de acompanhamento constante;

6. A responsabilidade pelo exame e seguimento dos RN em risco deve ser definida por cada unidade neonatal. Havendo necessidade de transferência de $\mathrm{RN}$ em acompanhamento para a ROP, a manutenção do acompanhamento periódico do prematuro precisa ser assegurada;

7. Após a alta, caso haja necessidade de acompanhamento oftalmológico, os pais precisam ser esclarecidos do risco de cegueira e que o seguimento na época adequada é fundamental para o sucesso do tratamento. Essa informação deve ser feita oralmente e por escrito;

8. Equipamentos necessários para o exame: oftalmoscópio indireto, lente de 28 ou 20 dioptrias, blefarostato e depressor escleral;

9. Dilatação: colírios de tropicamida $0,5 \%$ ou ciclopentolato $1 \%$ e fenilefrina $2,5 \%$. Uma gota de cada com intervalo de 5 minutos em cada olho 40 minutos antes do exame. Em caso de uso de blefarostato, instilar cloridrato de proparacaína $0,5 \%$.

\section{Tratamento}

1. Pela possibilidade de complicações anestésicas e oftalmológicas futuras deve ser obtida autorização dos pais/responsáveis para realizar o procedimento, utilizando-se um folheto informativo simples e de fácil compreensão;

2. Tratamento em até 72 horas está indicado:

a. Zona I: qualquer estágio com doença plus;

b. Zona I: estágio 3 sem plus;

c. Zona II: estágio 2 ou 3 com plus.

A doença limiar definida pelo CRYO-ROP não é mais determinante única para a decisão do tratamento ${ }^{(11)}$.

3. Midríase é necessária antes do procedimento;

4. Método recomendado: ablação da retina periférica avascular $360^{\circ}$, anterior a qualquer ROP, utilizando-se laser de diodo indireto e/ou crioterapia;

O procedimento deve ser realizado sob anestesia geral ou analgesia e sedação associadas à anestesia tópica e o paciente monitorizado por um neonatologista ou anestesista ${ }^{(18)}$. Pode ser realizado na unidade de terapia intensiva neonatal ou em centro cirúrgico, de acordo com a conveniência de cada centro.

A recuperação pós-tratamento deve ser feita na unidade neonatal de tratamento intensivo.

O tratamento medicamentoso pós-operatório consiste em uma combinação de antibiótico/esteróides tópicos por 7-10 dias.

A equipe de saúde envolvida nos cuidados dos prematuros, independentemente de terem sido submetidos a tratamento ou não, deve estar ciente que essas crianças apresentam uma maior chance de desenvolver problemas oftalmológicos tais como: estrabismo, ambliopia e erros refrativos ${ }^{(17)}$. O seguimento oftalmológico após a alta da unidade de terapia intensiva neonatal está sempre indicado.

O Brasil possui um programa de prevenção de ROP que está sendo implementado de forma progressiva a nível nacional. Faz-se necessário a conscientização mais ampla das equipes de saúde envolvidas - oftalmologistas e neonatologistas no sentido de expandir a implementação dos programas de triagem e prevenção da cegueira pela ROP. Só assim este programa poderá atingir o seu objetivo de reduzir incidência da cegueira e das outras complicações oftalmológicas da prematuridade extrema.

\section{ABSTRACT}

Retinopathy of prematurity is one of the main causes of childhood blindness. Worldwide, there are more than 50,000 children blind due to retinopathy of prematurity. Visual impairment is a consequence of retinal detachment. It can be detected by serial ophthalmologic examination of infants at risk, and those identified with the severe form of the disease can be treated by laser or cryotherapy, which can decrease significantly the blindness due to ROP. The Brazilian Society of Pediatrics, Brazilian Council of Ophthalmology and Brazilian Society of Pediatric Ophthalmology suggest a guideline for the detection and treatment of retinopathy of prematurity in Brazil. This document was based on the results of the I Workshop of Retinopathy of Prematurity and presents the attributes for the implementation of an efficient diagnostic and treatment program.

Keywords: Retinopathy of prematurity/diagnosis; Retinopathy of prematurity/surgery; Infant, premature; Practice guidelines

\section{REFERÊNCIAS}

1. Gilbert C, Rahi J, Eckstein M, O'Sullivan J, Foster A. Retinopathy of prematurity in middle-income countries. Lancet. 1997;350(9070):12-4.

2. Gilbert C, Fielder A, Gordillo L, Quinn G, Semiglia R, Visintin P, Zin A; International NO-ROP Group. Characteristics of infants with severe retinopathy of prematurity in countries with low, moderate, and high levels of development: implications for screening programs. Pediatrics. 2005;115(5): 518-25.

3. Darlow BA, Hutchinson JL, Simpson JM, Henderson-Smart DJ, Donoghue DA, Evans NJ. Variation in rates of severe retinopathy of prematurity among neonatal intensive care units in the Australian and New Zealand Neonatal Network. Br J Ophthalmol. 2005;89(12): 1592-6. Comment in: Br J Ophthalmol. 2005;89(12):1547.

4. An international classification of retinopathy of prematurity. Prepared by and International Committee. Br J Ophthalmol. 1984;68(10):690-7.

5. An international classification of retinopathy of prematurity. II. The classification of retinal detachment. The International Committee for the Classification of the Late Stages of Retinopathy of Prematurity. Arch Ophthalmol. 1987;105 (7):906-12.Erratum in: Arch Ophthalmol 1987;105(11):1498.

6. The International Classification of Retinopathy of Prematurity revisited. International Committee for the Classification of Retinopathy of Prematurity. Arch Ophthalmol. 2005;123(7):991-9. Comment in: Arch Ophthalmol. 2006;124 (11):1669-70.

7. Multicenter trial of cryotherapy for retinopathy of prematurity. Preliminary results. Cryotherapy for Retinopathy of Prematurity Cooperative Group. Arch Ophthalmol. 1988;106(4):471-9.

8. Multicenter trial of cryotherapy for retinopathy of prematurity. 3 1/2-year outcome - structure and function. Cryotherapy for Retinopathy of Prematurity Cooperative Group. Arch Ophthalmol. 1993;111(3):339-44.

9. Multicenter trial of cryotherapy for retinopathy of prematurity. Snellen visual 
acuity and structural outcome at 5 1/2 years after randomization. Cryotherapy for Retinopathy of Prematurity Cooperative Group. Arch Ophthalmol. 1996; 114(4):417-24.

10. Palmer EA, Hardy RJ, Dobson V, Phelps DL, Quinn GE, Summers CG, Krom CP, Tung B. Cryotherapy for Retinopathy of Prematurity Cooperative Group. 15-year outcomes following threshold retinopathy of prematurity: final results from the multicenter trial of cryotherapy for retinopathy of prematurity. Arch Ophthalmol. 2005;123(3):311-8.

11. Early Treatment for Retinopathy of Prematurity Cooperative Group. Revised indications for the treatment of retinopathy of prematurity: results of the early treatment for retinopathy of prematurity randomized trial. Arch Ophthalmol. 2003;121(12):1684-94. Comment in: Arch Ophthalmol. 2003;121(12):176971. Arch Ophthalmol. 2005;123(3):406-7; discussion 409-10. Arch Ophthalmol. 2005;123(3):406; discussion 409-10. Arch Ophthalmol. 2005;123(3):407-8; discussion 409-10. Arch Ophthalmol. 200;123(3):408-9; discussion 409-10.

12. Section on Ophthalmology American Academy of Pediatrics; American Academy of Ophthalmology; American Association for Pediatric Ophthalmology and Strabismus. Screening examination of premature infants for retinopathy of prematurity. Pediatrics. 2006;117(2):572-6. Erratum in: Pediatrics. 2006; 118(3):1324
13. Retinopathy of prematurity: guidelines for screening and treatment. The report of a Joint Working Party of The Royal College of Ophthalmologists and the British Association of Perinatal Medicine. Early Hum Dev. 1996;46(3):239-58.

14. Guidelines for screening examinations for retinopathy of prematurity. Canadian Association of Pediatric Ophthalmologists Ad Hoc Committee on Standards of Screening Examination for Retinopathy of Prematurity. Can J Ophthalmol. 2000;35(5):251-2; discussion 253-4.

15. Grupo Retinopatia da Prematuridade Brasil. Relatório do I Workshop Retinopatia da Prematuridade [texto na Internet]. Sociedade Brasileira de Pediatria. 2002. [citado 2007 Jan 12]. Disponível em: http://www.sbp.com.br/show_ item2.cfm?id_categoria=22\&id_detalhe $=1824 \&$ tipo_detalhes.

16. Gilbert CE, Rahi J, Quinn E. Visual impairment and blindness in children. In: Johnson GF, editor. Epidemiology of eye disease. New York: Chapman \& Hall; 1998.

17. Schalij-Delfos NE, de Graaf ME, Treffers WF, Engel J, Cats BP. Long term follow up of premature infants: detection of strabismus, amblyopia, and refractive errors. Br J Ophthalmol. 2000;84(9):963-7.

18. Haigh PM, Chiswick ML, O’Donoghue EP. Retinopathy of prematurity: systemic complications associated with different anaesthetic techniques at treatment. Br J Ophthalmol. 1997;81(4):283-7. 